Original Research

\title{
Distribution and Protection of Chinese Beech under the Background of Climate Change
}

\author{
Li Xiang ${ }^{1 \#}$, Hong Wang", Lei Liu², Haoxiang Zhao ${ }^{3}$, Yi Huang ${ }^{2}$, Hongmeng Chen ${ }^{2}$, \\ Yueqin $\mathrm{Ma}^{2}$, Ying $\mathrm{Mao}^{2}$, Liantong $\mathrm{Hu}^{2}$, Jinyao $\mathrm{Hu}^{2 *}$ \\ ${ }^{1}$ School of Economics, Southwest Minzu University, Chengdu, Sichuan, 610041, China \\ ${ }^{2}$ Engineering Research Center for Forest and Grassland Disaster Prevention and Reduction, \\ Mianyang Normal University, Mianyang, Sichuan, 621000, China \\ ${ }^{3}$ College of Geography and Environment Science, Northwest Normal University, Lanzhou, Gansu, 730070, China
}

Received: 15 July 2021

Accepted: 11 November 2021

\begin{abstract}
The biodiversity crisis caused by global warming is becoming more and more serious. Thus, many species are facing the crisis of survival, and some species are even on the verge of extinction. To scientifically evaluate the impact of global warming on the distribution, management and protection of Chinese Beech, the habitat suitability of 7 Chinese Beech species was simulated based on 853 literature distribution sites and 11 environmental factors under the current, 2070 RCP2.6 and 2070 RCP8.5 climate conditions and evaluated using ArcGIS 10.2 technology and the MaxEnt model. The results showed that (1) three environmental factors, namely, Precipitation of the Driest Quarter, Minimum Temperature of the Coldest Month and Mean Diurnal Range, were the key factors that influencing the distribution of Chinese Beech; (2) the total current area of suitable habitat of Chinese Beech was about $78615.60 \mathrm{~km}^{2}$, and only about $9527.36 \mathrm{~km}^{2}$ was located in protected areas (12.12\%); (3) with the continuous warming of the climate, the suitable habitat of Chinese Beech could lose up to $59.24 \%$, and the protected area would be decreasing continuously. Under the best and the most extreme climate scenarios for 2070, the suitable habitat area of Chinese Beech was reduced by $33386.90 \mathrm{~km}^{2}$ and $46573.50 \mathrm{~km}^{2}$, respectively, and the protected area decreased by $2,362.22 \mathrm{~km}^{2}$ and $5,056.40 \mathrm{~km}^{2}$, respectively. (4) The highly suitable habitat was projected to move the northwest and high altitude areas. Consequently, the protection of Chinese Beech is urgently required.
\end{abstract}

Keywords: climate change, Chinese Beech, MaxEnt, habitat suitability, protection, species distribution pattern

\# These authors contributed equally to this work.

*e-mail: jinyaohu123@163.com 


\section{Introduction}

In recent years, increasing attention has been focused on climate change, and studies have shown that climate is likely to rapidly change in only 10 years $[1,2]$. According to the Fifth Assessment Report of Intergovernmental Panel on Climate Change (IPCC), the global temperature in the $21^{\text {st }}$ century may increase by more than $1.5 \sim 2^{\circ} \mathrm{C}$ compared to that from $1850 \sim 1900$. The trend will continue to the end of the current century, with the last three decades warmer than any since 1850 . The main cause of global climate change is human activities [3]. From a regional scale perspective, climatic conditions are the leading factors determining species distribution [4]. Changes in temperature and precipitation caused by climate change will affect the distribution of suitable habitats for plants, resulting in regional migration and niche transfer of plants. The geographical distribution pattern of most species changes accordingly, which has an important impact on the entire ecosystem [5-7]. These adverse effects will be observed under future climate change. Therefore, species distribution models combined with GIS have been used to predict the change trend of suitable habitat and propose corresponding countermeasures [8,9].

Fagus is a tall deciduous genus of trees and widely distributed in the Pan-Arctic vegetation system. Fagus longipetiolata is the dominant species in temperate deciduous broad-leaved mixed forest $[10,11]$. The distribution area of the species of genus Fagus is mainly in east Asia, western Eurasia and eastern North America. In Europe and North America, due to its strong climatic suitability, Fagus has become climax vegetation [12]. Fagus species are an important forest resource and play an important role in water and soil conservation, biodiversity protection and land ecological security. These trees have high scientific research value and production potential. Meanwhile, Chinese Beech is one of the important research objects of flora, climate change and plant geography [13]. China has an extremely rich variety of plants in the Fagus genus. The study on the suitable habitat changes of the Chinese plants under the influence of climate change can provide evidence for the future distribution of North. In China, Fagus species are mainly distributed in the southern subtropical mountains, which have complex topographic and geological conditions and a mild and humid climate, and they present a discontinuous distribution pattern [12]. The classification of Chinese Beech is still not completely unified. Different scholars have divided the Beech genus into different species from different classification perspectives. However, it is generally accepted that there are about 10 to 14 species of Beech worldwide and 5 to 8 species in China [14-17]. The Chinese Beech species used in this paper include Fagus longipetiolata, Fagus hayatae, Fagus pashanica, Fagus engleriana, Fagus lucida, Fagus tientaiensis, and Fagus chienii. All species are endemic to China, and Fagus hayatae is a secondary protected plant in
China [18]. Based on the findings of these scholars, we believe that the distribution range of the genus of Chinese Beech may be significantly reduced in the future climate.

We conducted this study to explore the distribution pattern change and protection efficiency of the whole genus of Chinese Beech under the background of climate change. In this study, based on the geographical distribution data of the genus Chinese Beech, the MaxEnt model and ArcGIS tool were used to carry out habitat simulations and protection efficiency evaluations of the genus Chinese Beech.

\section{Materials and Methods}

\section{MaxEnt Model}

The MaxEnt model is a species distribution model (SDM), and it uses the known geographical distribution of species, combines the environmental factors of each location to conduct analyses and calculations, and finds the probability distribution with the maximum entropy as the optimal distribution to predict the adaptive region of species [19, 20]. This model is freely available on the website (http://www.cs.princeton.edu/ schapire/ maxent/). The MaxEnt model stands out among the many species distribution models due to its accuracy in prediction, operational stability and convenience of use. In recent years, the MaxEnt model has been widely used in the prediction of suitable habitats of tree species $[21,22]$. But previous studies are mostly limited to one or two species with more sensitive ecological niches, and few studies have focused on the dominant species and group building species [23].

\section{Species Distribution Data}

In this paper, species distribution records were obtained from the global biodiversity database (http://www.gbif.org/), the Chinese Virtual Herbarium (http://www.cvh.ac.cn/), field investigation and published literature [10-12, 24]. Because the distribution records of Chinese Beech in some literatures are very few and inaccurate, we conducted a field survey on its distribution in Sichuan to ensure the accuracy of the model. For each plant found, only one longitude and latitude coordinate per square kilometer was taken as an effective record. We investigated Nanjiang County of Bazhong City, Sichuan Province, Wanglang National Nature Reserve, Qingliangfeng National Nature Reserve, Qixingguan District and Nayong County of Bijie City, Guizhou Province, and made coordinates supplement for Chinese Beech.

To sort out the species distribution records collected for all specimens, documents and field surveys, the longitude and latitude are directly used as the species distribution data for species distribution records with longitude and latitude, whereas place names are searched 
based on a Google Earth map for species distribution records without longitude and latitude coordinates. If records of small geographical areas are available, then the longitude and latitude of these areas are used as the species distribution data; however, if these records are not available, then the longitude and latitude of the location of the village-level administrative center is used as the species distribution data. To improve the accuracy of the model, after repeated and invalid data are removed, the species distribution data are imported into ArcGIS. A buffer zone is built around these species distribution points with a kilometer radius. For species distribution points with buffer overlap, only one point is reserved. A total of 853 species distribution points are obtained. The species distribution points were obtained and saved in CSV format for use when running the model.

\section{Environmental Factors}

The environmental factors used in this paper can be divided into biological climate factors and topographic data. The bioclimatic factors are from the Worldclim database (http://worldclim.org/). Bioclimatic factors are derived from monthly temperature and rainfall to produce more biologically significant variables. These models are often used in species distribution modeling and related ecological modeling techniques. Bioclimatic factors represent annual trends, seasonality and extreme or limiting environmental factors (as shown in Table 1 from Biol to Bio19). The current climate data in the database were developed by Hijmans et al. 2005 [25]. The future climate data are the results of future climate simulation of the Intergovernmental Panel on Climate Change (IPCC) based on the Global Climate Model (GCM) under four Representative Concentration Paths (RCPs). The deviation correction is made according to the current climate of the global climate data Version 1.4. We downloaded global climate data Version 1.4 for current climate (1960a-1990a) and future climate 2070 (2061a-2080a). The future climate is based on data from the CCSM4 model and RCP2.6 and RCP8.5 scenarios provided by the IPCC/CMIP5. The scenarios with the lowest and highest $\mathrm{CO}_{2}$ emission concentrations are based on four typical concentration pathways (RCPs) [26]. In the RCP2.6 scenario, by the end of the $21^{\text {st }}$ century, the global average temperature will increase by $1^{\circ} \mathrm{C}$; and in the RCP 8.5 scenario, by the end of the $21^{\text {st }}$ century, the global temperatures will increase by an average of more than $2^{\circ} \mathrm{C}$. The spatial resolution of the bioclimate factor data was 30" (about $1 \mathrm{~km}$ ). Topographic data includes elevation data, slope data and aspect data. Elevation data from the national aeronautics and space administration released the global digital elevation model (http://srtm.csi.cgiar.org/) at a resolution of $90 \mathrm{~m}$. Slope data and aspect data were extracted from elevation data with a resolution of $90 \mathrm{~m}$. A total of 22 environmental factors were collected. (Table 1).
Table 1. Environmental variables.

\begin{tabular}{|c|c|}
\hline Code & Environmental Variable \\
\hline Bio1 & Annual Mean Temperature \\
\hline Bio2 & Mean Diurnal Range \\
\hline Bio3 & Isothermality \\
\hline Bio4 & Temperature Seasonality \\
\hline Bio5 & Max Temperature of Warmest Month \\
\hline Bio6 & Min Temperature of Coldest Month \\
\hline Bio7 & Temperature Annual Range \\
\hline Bio8 & Mean Temperature of Wettest Quarter \\
\hline Bio9 & Mean Temperature of Driest Quarter \\
\hline Bio10 & Mean Temperature of Warmest Quarter \\
\hline Bio11 & Mean Temperature of Coldest Quarter \\
\hline Bio12 & Annual Precipitation \\
\hline Bio13 & Precipitation of Wettest Month \\
\hline Bio14 & Precipitation of Driest Month \\
\hline Bio15 & Precipitation Seasonality (Coefficient of Variation) \\
\hline Bio16 & Precipitation of Wettest Quarter \\
\hline Bio17 & Precipitation of Driest Quarter \\
\hline Bio18 & Precipitation of Warmest Quarter \\
\hline Bio19 & Precipitation of Coldest Quarter \\
\hline Dem & Elevation \\
\hline Slope & Slope \\
\hline Aspect & Aspect \\
\hline
\end{tabular}

\section{Boundary Data of the Study Area}

China's boundary data are based on the 1:400,000 basic vector data for China from the national center for basic geographic information (http://www.ngcc.cn/).

\section{Protected Area Data}

Boundary data of for the national nature reserves were obtained from the Resource and Environmental Science Data Center of the Chinese Academy of Sciences (http://www.resdc.cn).

\section{Processing of Species Distribution Data}

\section{Environmental Factor Processing}

The ArcGIS 10.2 projection tool (http://www.esri. com/software/arcgis/) was used for all environmental factor (including bioclimatic factor data and topographic factor data), and the boundary vector data of China's 1:400,000 scale are projected and unified based on the WGS1984 coordinate system. All environmental factors 
were resampled using the ArcGIS 10.2 resampling tool, and all environmental factor had a uniform spatial scale with a unified resolution of 30 " (about $1 \mathrm{~km}$ ).

Using the ArcGIS 10.2 clipping tool, all environmental factor (including bioclimate factor data and topographic data) were clipped according to the boundary vector data of China's 1:400,000 scale so that they had a uniform boundary.Then, slope data and aspect data were extracted from processed elevation data by using the slope extraction tool and aspect extraction tool in ArcGIS 10.2. The slope and aspect data will maintain the same coordinate system, spatial scale and boundaries as the processed elevation data. In this study, we reclassified the aspect data through ArcGIS 10.2 so that the value range of the slope data was an integer between 0 and 8 . Each value represents one direction, where 0 is flat without slope and values 1-8 correspond to due north, northeast, east, southeast, south, southwest, west and northwest.

\section{Factor Filtering Performed for the Model}

Correlations are observed among environmental factors, and these correlations will have a certain impact on the simulation results of the model [27]. Therefore, to improve the accuracy of the prediction results of the model, 22 environmental factors need to be screened. First, all the environmental factors values of each species distribution point were extracted by the Extraction tool in ArcGIS. Each point is extracted to obtain 22 values, and the saved values are export as a .CSV file. Then, $\mathrm{R}$ software is used to conduct a correlation analysis on the environmental factor values of all species distribution points, and the results are shown in Table 2.

For two factors whose absolute value of correlation exceeds 0.8 , one factor is selected and the remaining environmental factors are considered to have a low correlation [28]. Based on the constraints of extreme weather on species, the environmental factors that remain are Bio2, Bio3, Bio5, Bio6, Bio7, Bio8, Bio14, Bio15, Bio16, Bio17, aspect, and slope. Finally, ArcGIS 10.2 is used to convert the filtered environmental factors into ASCII format, and the species distribution data in CSV format were imported into MaxEnt 3.4.1, and the model was then run.

\section{Parameter Settings}

Each tree species was predicted under different climatic conditions. All model parameters remain the same. For the imported environmental factors, the aspect was selected as the classified data type and the rest were classified as the continuous data type. All model features were checked with the linear feature, quadratic equation feature, product feature, key feature and automation feature. The random test percentage was $25 \%$, the regularization multiple was 1 , the maximum iteration number was 5000, the maximum background value was 10000 , and the repetition number was 10 . The verification method was cross validation. The other values were set to default values.

\section{Model Accuracy Evaluation}

A receiver operating characteristic curve (ROC) was generated for all models. The accuracy of the model was measured by the area under the receiver operator curve (AUC) [29]. The ROC curve was formed by taking the false positive rate as the abscissa and the true positive rate as the ordinate. The closed area formed by the ROC curve and abscissa was the AUC. The higher the value of AUC, the more accurate the model will be. The evaluation criteria of the AUC value were as follows: AUC between 0.5 and 0.6, fail; 0.6 0.7, poor; 0.7 0.8, fair; 0.8 0.9, good; and 0.9 1.0, excellent [30]. Jackknife was used to analyze the single factor contribution rate. The output model results were in logical value format, in which the output was the probability value of species distribution $(0 \sim 1)$. The output file format was ASCII.

\section{Suitable Habitat Division and Hot Spots}

The operation results of MaxEnt include the potential distribution simulation diagram of each species in the current and future climate scenarios [23, 31]. The value represents the probability of species distribution. The larger the distribution probability value, the higher the habitat suitability. To make the results more reliable, this study deleted the suitable habitat with occurrence probabilities lower than 0.75 , and only the highly suitable habitats with an occurrence probability higher than 0.75 were analyzed and discussed. In addition, ArcGIS 10.2 was used to conduct a spatial superposition analysis on the results of various species of Chinese Beech under the same climate conditions. By combining the highly suitable habitats of 7 species under the same climatic context, the highly suitable habitat distribution of Chinese Beech was obtained. The intersection tool in ArcGIS was used to obtain the intersection of three highly suitable habitats as the hot spot distribution area of Chinese Beech.

\section{Changes in Suitable Habitats}

The distribution of suitable habitats is often irregular, and the boundary is not easily determined. Therefore, we use the change of the geometric centroid of suitable habitat in different scenarios to represent the change direction and change distance of the suitable habitat of the genus Chinese Beech under the condition of climate change. The geometric centroid suitable for habitat was found by calculating the longitude and latitude of geometric centroid in the ArcGIS10.2 attribute table [28, 29]. 


\begin{tabular}{|c|c|c|c|c|c|c|c|c|c|c|c|c|c|c|c|c|c|c|c|c|c|c|c|c|c|c|}
\hline$\frac{0}{\frac{0}{n}}$ & & & & & & & & & & & & & & & & & & & & & & & & & & $\stackrel{8}{-}$ \\
\hline छี & & & & & & & & & & & & & & & & & & & & & & & & & $\stackrel{8}{-}$ & 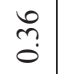 \\
\hline 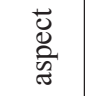 & & & & & & & & & & & & & & & & & & & & & & & & $\underset{-}{\stackrel{\leftrightarrow}{-}}$ & $\begin{array}{l}\stackrel{0}{0} \\
\stackrel{\varphi}{1}\end{array}$ & $\stackrel{t}{0}$ \\
\hline$\stackrel{\circ}{\stackrel{\circ}{n}}$ & & & & & & & & & & & & & & & & & & & & & & & $\underset{-}{\stackrel{8}{1}}$ & $\begin{array}{l}\infty \\
0 \\
0\end{array}$ & 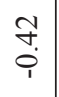 & 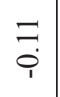 \\
\hline$\stackrel{\infty}{\stackrel{\infty}{n}}$ & & & & & & & & & & & & & & & & & & & & & $£$ & 足 & $\begin{array}{l}7 \\
0 \\
0\end{array}$ & $\underset{8}{8}$ & $\stackrel{7}{\overparen{0}}$ & $\stackrel{0}{\circ}$ \\
\hline$\stackrel{\overline{0}}{\frac{0}{n}}$ & & & & & & & & & & & & & & & & & & & & \& & ? & 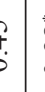 & \begin{tabular}{l}
$\infty$ \\
$\infty$ \\
\hdashline \\
0
\end{tabular} & $\stackrel{0}{0}$ & $\begin{array}{l}\stackrel{9}{+} \\
\stackrel{1}{1}\end{array}$ & $\stackrel{0}{\stackrel{1}{i}}$ \\
\hline $\begin{array}{l}0 \\
\stackrel{0}{0}\end{array}$ & & & & & & & & & & & & & & & & & & & $\stackrel{\&}{-}$ & a & $\begin{array}{l}* \\
\infty \\
0\end{array}$ & 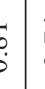 & $\begin{array}{l} \pm \\
\vdots \\
0\end{array}$ & $\begin{array}{l}\stackrel{0}{0} \\
\dot{0}\end{array}$ & $\frac{ \pm}{\dot{0}}$ & $\begin{array}{l}0 \\
\stackrel{0}{i} \\
\dot{\varphi}\end{array}$ \\
\hline$\frac{n}{\stackrel{\circ}{n}}$ & & & & & & & & & & & & & & & & & & $\stackrel{8}{-}$ & $\begin{array}{l}\text { त̂. } \\
\text { }\end{array}$ & $\stackrel{\infty}{2}$ & $\begin{array}{l} \pm \\
⿱ \\
\end{array}$ & 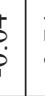 & $\begin{array}{c}+ \\
\stackrel{i}{i} \\
\end{array}$ & $\begin{array}{l}0 \\
0 \\
0 \\
i\end{array}$ & $\stackrel{\text { I. }}{\circ}$ & $\stackrel{8}{\circ}$ \\
\hline 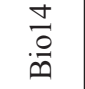 & & & & & & & & & & & & & & & & & $\underset{-}{8}$ & $\stackrel{\hat{\imath}}{\stackrel{i}{i}}$ & $\begin{array}{l}\infty \\
0 \\
0\end{array}$ & 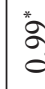 & I & $\dot{s}$ & :̊ & $\begin{array}{l}\infty \\
\stackrel{0}{0}\end{array}$ & $\begin{array}{l}\infty \\
\stackrel{\infty}{1} \\
\stackrel{i}{1}\end{array}$ & $\begin{array}{l}\text { oे } \\
\text { i. }\end{array}$ \\
\hline$\frac{m}{\circ}$ & & & & & & & & & & & & & & & 8 & & $\begin{array}{l}\infty \\
\stackrel{\infty}{0} \\
0\end{array}$ & $\begin{array}{l}\stackrel{+}{4} \\
\stackrel{i}{i}\end{array}$ & $\begin{array}{l}* \\
\stackrel{0}{\circ}\end{array}$ & a & $\begin{array}{c}* \\
\infty \\
0\end{array}$ & : & $\stackrel{+}{\stackrel{0}{0}}$ & $\stackrel{8}{\circ}$ & $\begin{array}{l}0 \\
\stackrel{-}{i} \\
\end{array}$ & $\begin{array}{l}0 \\
\stackrel{0}{0} \\
\stackrel{1}{1}\end{array}$ \\
\hline$\stackrel{\cong}{\stackrel{0}{0}}$ & & & & & & & & & & & & & & $\stackrel{8}{-}$ & $\mid \begin{array}{l}a \\
0 \\
o\end{array}$ & & $\begin{array}{l}\infty \\
\infty \\
\infty \\
\infty \\
0\end{array}$ & है & 。̊̊ & $\begin{array}{l}\ddot{a} \\
\ddot{0}\end{array}$ & $\stackrel{2}{0}$ & $?$ & $\vec{a}$ & $\stackrel{0}{\circ}$ & 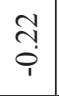 & $\begin{array}{l}\text { o̊ } \\
\stackrel{i}{i}\end{array}$ \\
\hline $\overrightarrow{\overline{0}}$ & & & & & & & & & & & & & $\stackrel{8}{-}$ & శె & $\stackrel{f}{0}$ & & तै & $\stackrel{\circ}{\circ}$ & $\stackrel{\text { In }}{0}$ & ర్ల & ฮี & $?$ & 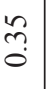 & $\stackrel{0}{0}$ & $\begin{array}{l}\hat{0} \\
\dot{\varphi}\end{array}$ & 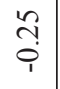 \\
\hline$\frac{0}{\circ}$ & & & & & & & & & & & & $\stackrel{8}{-}$ & $\stackrel{?}{\circ}$ & $\frac{9}{0}$ & $\frac{\infty}{0}$ & & $\bar{m}$ & $\begin{array}{l}\hat{n} \\
\stackrel{i}{i}\end{array}$ & $\stackrel{\infty}{-0}$ & $\hat{a}$ & $\begin{array}{l}8 \\
0 \\
1\end{array}$ & $\dot{s}$ & 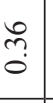 & $\stackrel{\infty}{0}$ & $\begin{array}{l}n \\
\stackrel{n}{2} \\
\grave{i}\end{array}$ & $\begin{array}{c}\text { m} \\
\stackrel{i}{i}\end{array}$ \\
\hline$\stackrel{\circ}{\circ}$ & & & & & & & & & & & $\stackrel{8}{.}$ & $\stackrel{n}{0}$ & 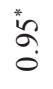 & $\stackrel{\text { f }}{0}$ & ț & & 宅 & $\underset{i}{ \pm}$ & ñ & fo & $\stackrel{m}{o}$ & $?$ & గñ & $\hat{0}$ & $\begin{array}{l}\infty \\
0 \\
\dot{\varphi} \\
i\end{array}$ & $\begin{array}{l}\hat{n} \\
\text { ọ }\end{array}$ \\
\hline$\stackrel{\infty}{\stackrel{\oplus}{\varphi}}$ & & & & & & & & & $\stackrel{8}{-}$ & & $\begin{array}{c}\tilde{n} \\
\stackrel{0}{0}\end{array}$ & $\begin{array}{l}\hat{\imath} \\
\dot{0}\end{array}$ & ্ָర & i. & o̊ & & $\begin{array}{l}\text { do } \\
\stackrel{0}{i}\end{array}$ & $\stackrel{\infty}{\circ}$ & $\begin{array}{l}\text { oे } \\
\text { i }\end{array}$ & $\begin{array}{l}0 \\
0 \\
0\end{array}$ & $\begin{array}{l}\delta \\
0 \\
i\end{array}$ & $\vec{b}$ & $\begin{array}{l}\stackrel{0}{0} \\
\stackrel{1}{1} \\
\end{array}$ & $\begin{array}{c}\mathrm{o} \\
\dot{0}\end{array}$ & $\begin{array}{l}\stackrel{R}{2} \\
i \\
i\end{array}$ & $\begin{array}{l}\hat{n} \\
\stackrel{i}{i}\end{array}$ \\
\hline$\hat{\mathscr{a}}$ & & & & & & & & $\stackrel{8}{\circ}$ & $\frac{\infty}{0}$ & & 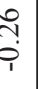 & $\hat{n}$ & 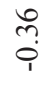 & กֶ. & $\hat{i}$ & & 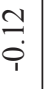 & $\stackrel{\infty}{\stackrel{\infty}{i}}$ & लें & $\begin{array}{l}0 \\
0 \\
0 \\
1\end{array}$ & in & $?$ & 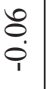 & $\stackrel{0}{0}$ & $\begin{array}{l}\tilde{3} \\
\stackrel{1}{1}\end{array}$ & $\begin{array}{l}\text { oे } \\
\text { i. }\end{array}$ \\
\hline$\stackrel{0}{\circ}$ & & & & & & & $\stackrel{\&}{-}$ & के & $\stackrel{\overbrace{}}{0}$ & & $\hat{\sigma}$ & $\vec{r}$ & 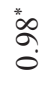 & $\stackrel{+}{m}$ & $\stackrel{?}{?}$ & 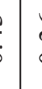 & $\begin{array}{l}0 \\
? \\
0 \\
0\end{array}$ & $\begin{array}{l}\infty \\
\stackrel{0}{0} \\
\stackrel{0}{1}\end{array}$ & $\stackrel{f}{0}$ & $\stackrel{J}{0}$ & $\tilde{3}$ & $?$ & 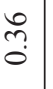 & $\hat{0}$ & $\begin{array}{l}0 \\
0 \\
i \\
i\end{array}$ & 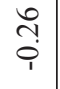 \\
\hline$\stackrel{n}{\infty}$ & & & & & & 8 & $\begin{array}{l}\text { to } \\
\stackrel{0}{\circ}\end{array}$ & $\stackrel{0}{0}$ & $\stackrel{?}{\stackrel{0}{0}}$ & & $\begin{array}{l}0 \\
0 \\
0 \\
\dot{0}\end{array}$ & $\stackrel{*}{\circ}$ & $\begin{array}{l}n \\
0 \\
0\end{array}$ & 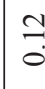 & $\frac{0}{0}$ & & \begin{tabular}{l}
$\tilde{2}$ \\
\hdashline \\
0
\end{tabular} & $\begin{array}{l}\hat{n} \\
\stackrel{1}{1}\end{array}$ & $\stackrel{\circ}{\circ}$ & $\hat{\tilde{z}}$ & $\frac{\infty}{0}$ & 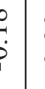 & \begin{tabular}{l}
$\stackrel{0}{0}$ \\
\hdashline \\
0
\end{tabular} & $\begin{array}{l}8 \\
0 \\
0\end{array}$ & 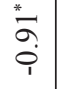 & $\begin{array}{c}\text { m} \\
\stackrel{i}{i}\end{array}$ \\
\hline$\stackrel{+}{\stackrel{9}{0}}$ & & & & $\varepsilon$ & & f. & $\begin{array}{c}\text { חे } \\
\text { ?̦ }\end{array}$ & ث̊ & ָָ & 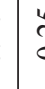 & 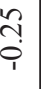 & F. & 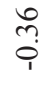 & $\frac{m}{9}$ & ஸे & & 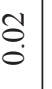 & 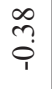 & $\begin{array}{c}m \\
? \\
?\end{array}$ & $\hat{a}$ & in & $\begin{array}{l}y_{0}^{\prime} \\
? \\
?\end{array}$ & $\stackrel{n}{0}$ & $\begin{array}{c}\tilde{O} \\
\stackrel{0}{0}\end{array}$ & 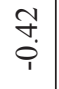 & $\begin{array}{l}\vec{\vdots} \\
\vec{i}\end{array}$ \\
\hline$\stackrel{\tilde{\theta}}{0}$ & & & $\stackrel{8}{-}$ & ? & & $\begin{array}{l}5 \\
\\
\end{array}$ & $\stackrel{\text { ñ }}{0}$ & $\begin{array}{l}0 \\
\text { in } \\
\text { in }\end{array}$ & $\tilde{0}$ & & ָે̀ & $\begin{array}{l}n \\
\\
i\end{array}$ & ợ & $\begin{array}{l}\hat{a} \\
\hat{i}\end{array}$ & $\frac{1}{0}$ & 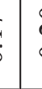 & 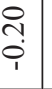 & $\vec{\sigma}$ & $\frac{\partial}{0}$ & $\begin{array}{l}\tilde{Z} \\
\text { Q }\end{array}$ & $\stackrel{+}{\tilde{c}}$ & ? & $\begin{array}{l}2 \\
\dot{0} \\
1\end{array}$ & $\begin{array}{l}\overrightarrow{0} \\
\dot{0} \\
1\end{array}$ & $\stackrel{m}{0}$ & $\stackrel{\circ}{\circ}$ \\
\hline$\stackrel{\widetilde{\theta}}{\circ}$ & & $\stackrel{8}{-}$ & $\underset{S}{0}$ & $\hat{\varepsilon}$ & & \pm & $\begin{array}{l}\stackrel{2}{0} \\
\stackrel{1}{1}\end{array}$ & $\bar{m}$ & $\stackrel{m}{0}$ & & $\begin{array}{l}8 \\
0 \\
0\end{array}$ & $\begin{array}{l}n \\
0 \\
0\end{array}$ & $\stackrel{0}{\circ}$ & J. & 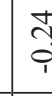 & 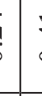 & $\begin{array}{l}\vec{F} \\
\dot{0} \\
\dot{0}\end{array}$ & $\tilde{n}$ & $\begin{array}{c}\text { ț } \\
\stackrel{1}{1}\end{array}$ & ले & $\hat{\tilde{\hat{z}}}$ & 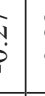 & $\begin{array}{l}m \\
\dddot{n} \\
i\end{array}$ & $\begin{array}{l}8 \\
0\end{array}$ & $=$ & $\underset{\delta}{0}$ \\
\hline$\vec{\circ}$ & $\stackrel{8}{-}$ & $\stackrel{\infty}{0}$ & $\stackrel{0}{\circ}$ & \& & & '. & ڤু & $\begin{array}{l}2 \\
0 \\
i\end{array}$ & $\stackrel{n}{\approx}$ & & $\stackrel{a}{o}$ & $\vec{a}$ & ๙ૂ. & กิ & $\stackrel{t}{3}$ & 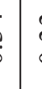 & $\stackrel{m}{3}$ & $\frac{7}{i}$ & $\stackrel{n}{m}$ & $\tilde{z}$ & $\frac{m}{0}$ & ? & $\hat{m}$ & $\begin{array}{l}\infty \\
\stackrel{0}{0}\end{array}$ & $\begin{array}{l}{ }_{0}^{*} \\
\infty \\
\dot{\varphi}\end{array}$ & $\overrightarrow{\tilde{̣}}$ \\
\hline 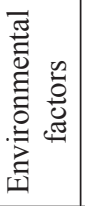 & $\overrightarrow{0}$ & $\tilde{c}$ & $\tilde{\rho}$ & t) & & $\frac{\hat{\theta}}{m}$ & $\stackrel{\circ}{\stackrel{\oplus}{9}}$ & $\hat{9}$ & $\stackrel{\infty}{\infty}$ & & 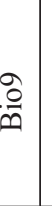 & $\frac{\circ}{\circ}$ & $\bar{\Xi}$ & $\frac{7}{9}$ & $\frac{2}{\infty}$ & t. & $\begin{array}{l}\vec{\Delta} \\
\stackrel{\circ}{\oplus}\end{array}$ & $\frac{n}{0}$ & $\begin{array}{l}0 \\
0 \\
0\end{array}$ & $\frac{7}{9}$ & $\frac{\infty}{\infty}$ & $\begin{array}{l}0 \\
\vdots \\
0 \\
b\end{array}$ & $\stackrel{\overrightarrow{0}}{\frac{0}{0}}$ & 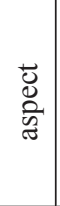 & छี & $\frac{\ddot{a}}{n}$ \\
\hline
\end{tabular}




\section{Protection Efficiency Assessment}

In this study, the ratio of the area of national nature reserves occupied by highly suitable areas under various climatic conditions and their corresponding highly suitable areas is taken as the protection rate, and this value is used to evaluate the efficiency of species protection [32].

\section{Results and Discussion}

\section{Accuracy Evaluation of Model Simulation}

After running MaxEnt, the distribution maps of suitable areas for Chinese Beech under the current and future climate scenarios were obtained. MaxEnt also generates distribution results of the maximum, minimum, median and mean values of each species. The subsequent processing and analysis in this study used the mean distribution results of the model operation. Under the $25 \%$ cross validation, the average AUC values for the training set after repeating the calculations 10 times were higher than 0.9 . The highest value was 0.994 , and the lowest was 0.942 , indicating that the accuracy of the model was relatively high. The contribution rate of 11 environmental factors was analyzed by Jackknife test. The AUC value of each species and the contribution rate of each environmental factor are shown in Table 3.

The MaxEnt model has been widely used in recent years. The accuracy of this model has been widely recognized. Moreover, it is convenient to use. Only species distribution point data and environmental information of the corresponding research area are needed to obtain more accurate results, and such functionality is lacking in other species distribution models, especially when the fewer sample data are available; in addition, the predictions of the MaxEnt model are better than that of other models [33-35]. This study was based on a total of 853 points and 11 environmental factors for 7 Chinese Beech species. The average AUC values of the training set obtained after 10 repeated calculated under $25 \%$ cross validation were all higher than 0.9 , indicating high credibility among the research results.

\section{Dominant Limiting Environmental Factors}

As shown in Table 3, the dominant limiting environmental factors depend on species. Fagus longipetiolata was mainly affected by three environmental factors: driest seasonal precipitation (Bio17), wettest seasonal precipitation (Biol6) and coldest monthly minimum temperature (Bio6); Fagus hayatae was mainly affected by three environmental factors: the driest seasonal precipitation (Bio17), the mean daily temperature range (Bio2) and the hottest monthly maximum temperature

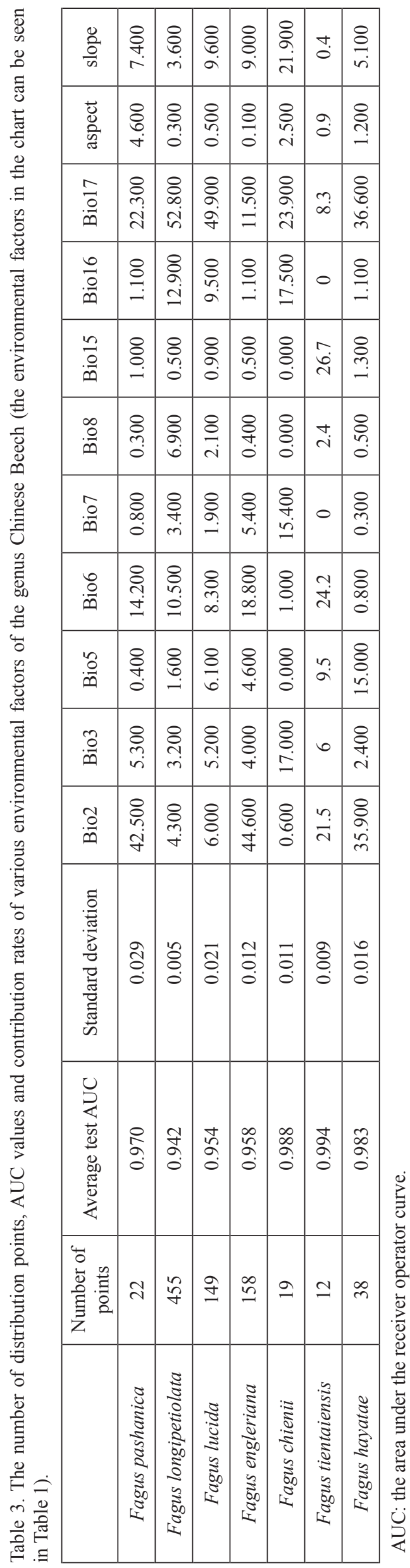


(Bio5); Fagus pashanica was mainly affected by three environmental factors: average daily temperature range (Bio2), driest quarterly precipitation (Bio17) and coldest monthly minimum temperature (Bio6); Fagus engleriana was mainly affected by three environmental factors: average daily temperature range (Bio2), lowest monthly temperature (Bio6) and driest quarterly precipitation (Bio17); Fagus lucida was mainly affected by three environmental factors: driest season precipitation (Bio17), slope (slop) and wettest season precipitation (Bio16); Fagus tientaiensis was mainly affected by three environmental factors, namely, precipitation seasonal variation coefficient (Bio15), lowest temperature in the coldest month (Bio06) and average daily temperature range (Bio2); and Fagus chienii was mainly affected by driest season precipitation (Bio17), slope (slop) and wettest season precipitation (Biol6).

Plenty of studies have demonstrated that temperature and precipitation are key factors that determine species ranges $[5,36,37]$. In the present study, according to the contribution rate and frequency, the three environmental factors driest season precipitation (Bio17), coldest month minimum temperature (Bio6) and average daily temperature range (Bio2) were the dominant environmental factors that restrict the distribution of species within the genus Chinese Beech. Likewise, Fang et al. found that the growing season temperature regime was the most important climatic factor affecting the geographical distribution of Chinese Beech in China, and the precipitation was the key factor limiting its distribution in the northern boundary [38].

\section{Highly Suitable Habitat under Various Climatic Conditions}

ArcGIS 10.2 was used to merge all 7 species in the current and 2070 RCP2.6 and RCP8.5 climate scenarios, and the high potential areas obtained by the model calculations were merged. Highly suitable habitats in China were observed under the current and 2070 RCP2.6 and RCP8.5 climate and environmental conditions for Chinese Beech (Figs 1-3).

The current highly suitable habitat of Chinese Beech was mainly located at the junction of Sichuan and Gansu, Shaanxi, Chongqing and Hubei provinces and in the south-central and northeast parts of Sichuan Province. Moreover, these areas scattered in Guizhou, Hunan and Guangxi provinces, along the Guangdong province border area, and in eastern Zhejiang province in southern China, and small distribution areas were observed in northeastern Yunnan province, and Taiwan, Jiangxi, Fujian and Anhui provinces. The total distribution area was about $78615.60 \mathrm{~km}^{2}$.

Under the RCP2.6 climate condition in 2070, the highly suitable habitat of Chinese Beech is mainly located in the south-central and northeastern part of Sichuan province, the junction of Sichuan with Gansu, Shanxi and Chongqing, and the western part of Hubei province. Moreover, these areas area scattered in the borderland of Guizhou, Hunan, Guangxi and Guangdong, the northeast of Yunnan province, the junction of Zhejiang and Fujian provinces, and the junction of Anhui and Hubei provinces, and a small distribution is observed in Jiangxi province. It was worth

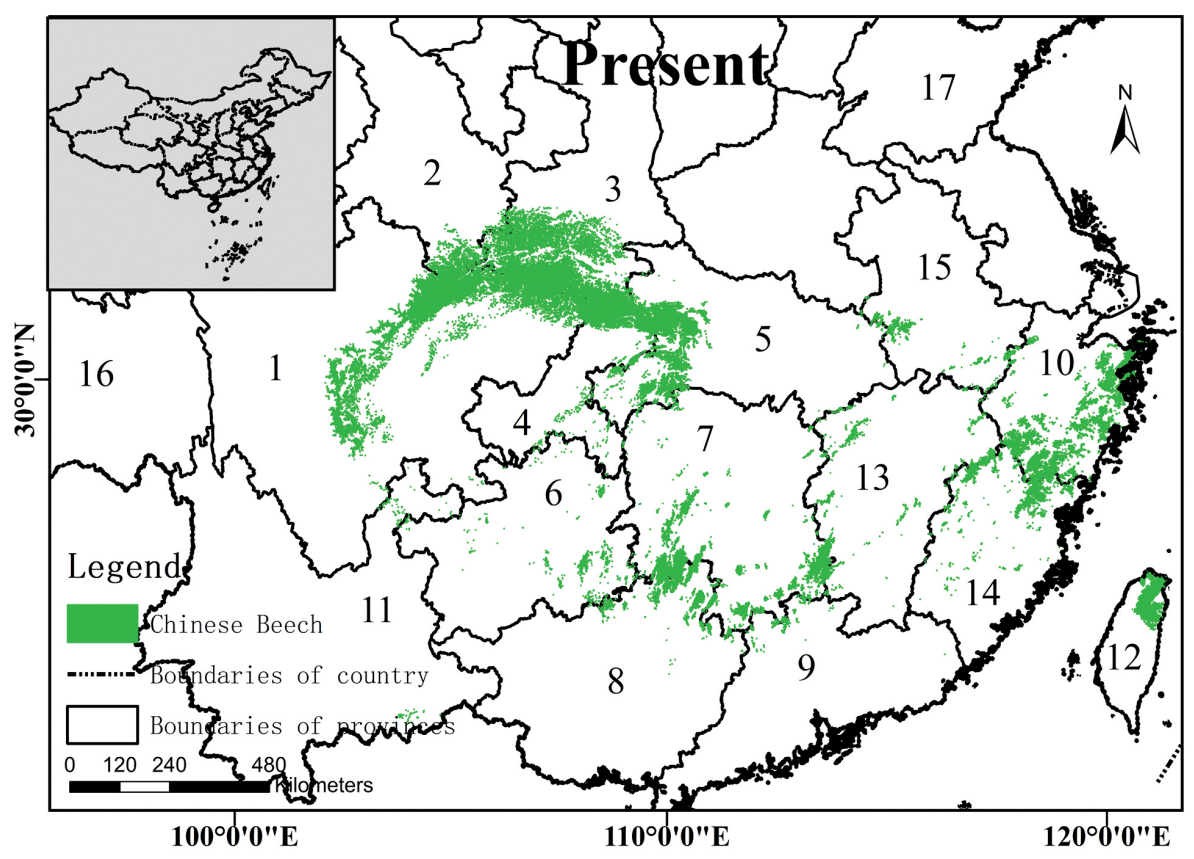

Fig. 1. Highly suitable habitat of 7 species of Chinese Beech under the current climate (1, Sichuan; 2, Gansu; 3, Shaanxi; 4, Chongqing; 5, Hubei; 6, Guizhou; 7, Hunan; 8 Guangxi; 9 Guangdong; 10 Zhejiang; 11, Yunnan; 12, Taiwan; 13, Jiangxi; 14, Fujian; 15, Anhui; 16, Tibet; 17, Shandong.) 


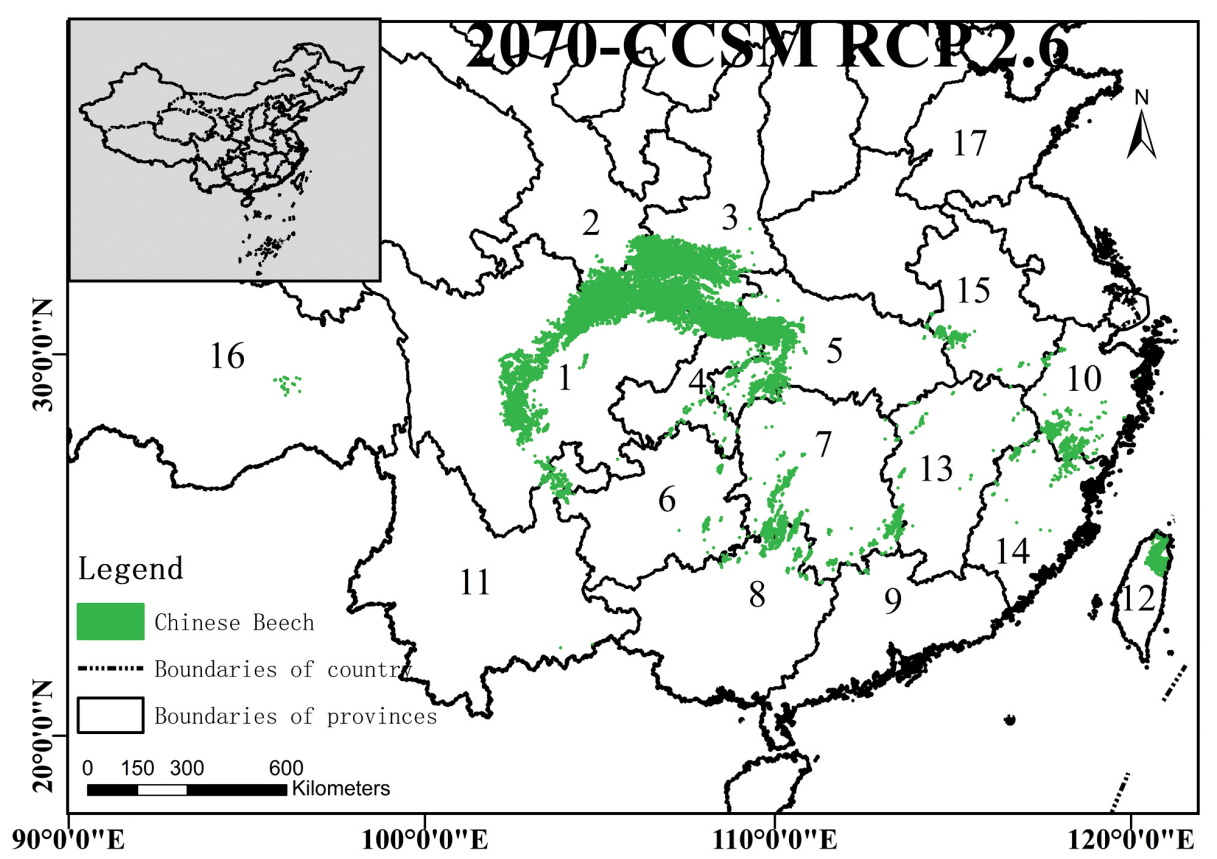

Fig. 2. Highly suitable habitat of 7 species of Chinese Beech under the RCP2.6 climate scenario in 2070 (1, Sichuan; 2, Gansu; 3 , Shaanxi; 4, Chongqing; 5, Hubei; 6, Guizhou; 7, Hunan; 8 Guangxi; 9 Guangdong; 10 Zhejiang; 11, Yunnan; 12, Taiwan; 13, Jiangxi; 14, Fujian; 15, Anhui; 16, Tibet; 17, Shandong.)

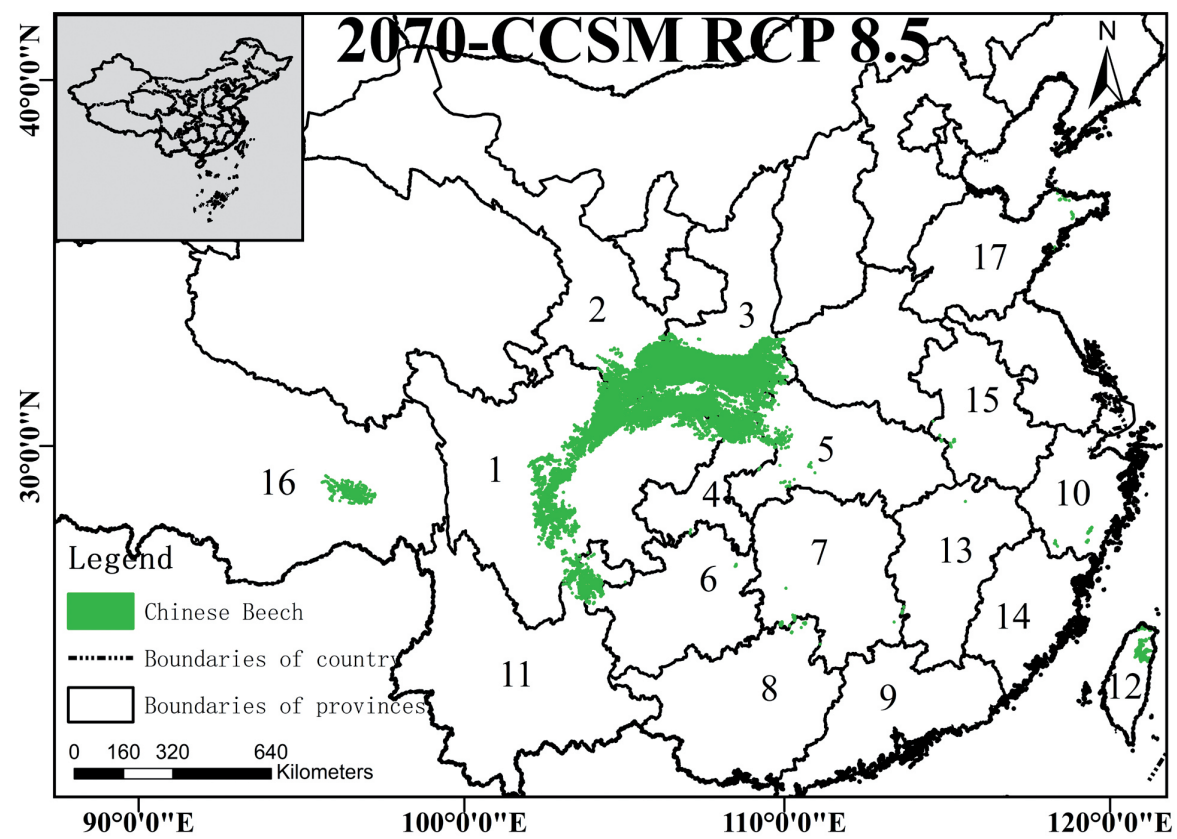

Fig. 3. Highly suitable habitat of 7 species of Chinese Beech under the RCP8.5 climate scenario in 2070 (1, Sichuan; 2, Gansu; 3 , Shaanxi; 4, Chongqing; 5, Hubei; 6, Guizhou; 7, Hunan; 8 Guangxi; 9 Guangdong; 10 Zhejiang; 11, Yunnan; 12, Taiwan; 13, Jiangxi; 14, Fujian; 15, Anhui; 16, Tibet; 17, Shandong.)

noting that a new highly suitable habitat appears in the eastern region of Tibet under this climate condition. The total distribution area was about $45228.70 \mathrm{~km}^{2}$.

Under the RCP8.5 climate condition in 2070, the highly suitable habitat of Chinese Beech was mainly concentrated in northeast Sichuan province, south-central and southern China, south Shaanxi province, southeast Gansu province, north Chongqing municipality, northwest Hubei province, northeast Yunnan province and eastern Tibet. Moreover, these areas area scattered in Taiwan, Guangxi, Guangdong, Hunan, Jiangxi, Zhejiang and Anhui, and a small distribution is observed in northeast Shandong province. The total distribution area was about $32,042.10 \mathrm{~km}^{2}$. 


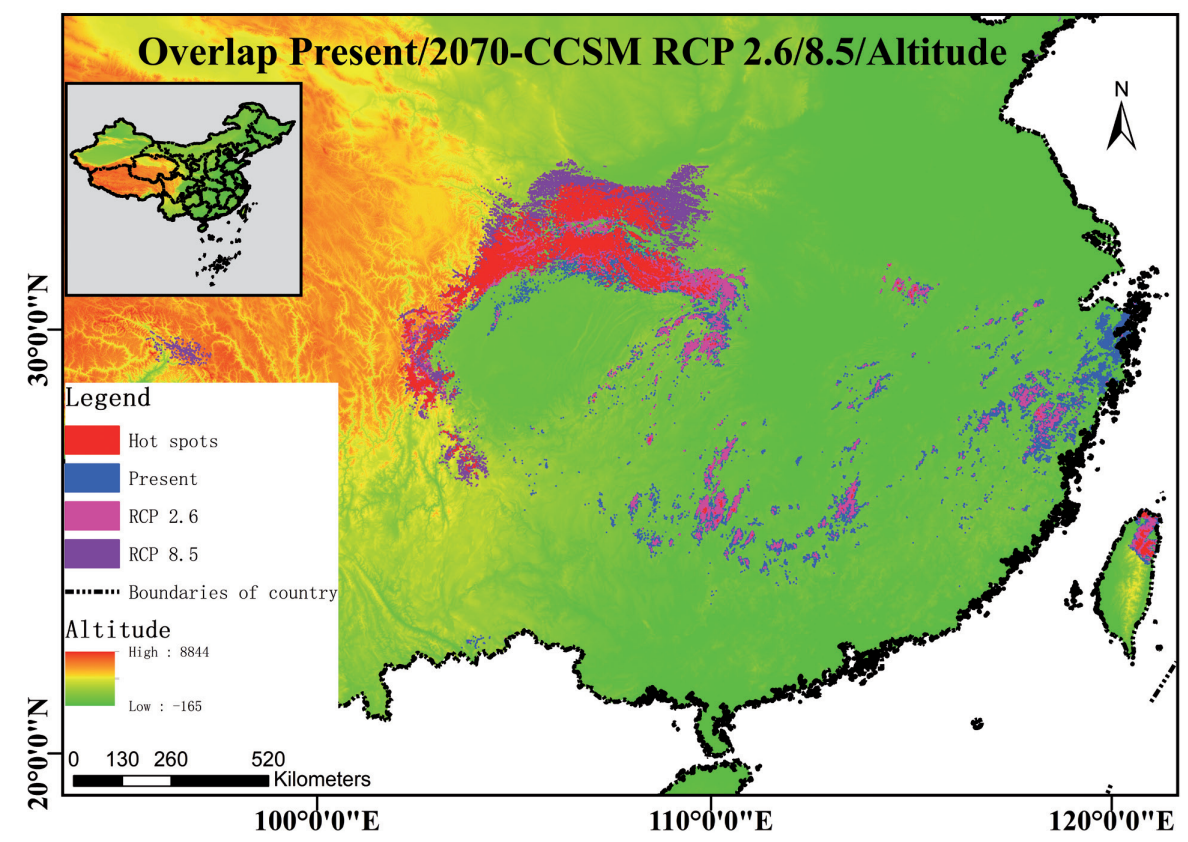

Fig. 4. Suitable habitat and altitude of 7 species of Chinese Beech under different climatic conditions.

\section{Highly Suitable Habitat Change}

A superposition analysis was performed as shown in Fig. 4. Northeast and south-central Sichuan province, south Shaanxi Province, southeast Gansu province, north Chongqing municipality, and northwest Hubei province are the hot spots of Chinese Beech. In addition, compared with the current climate situation, the area of highly suitable habitat of Chinese Beech is decreasing in the future climatic environment (Table 4). Under RCP2.6, the area decreased by about $33386.90 \mathrm{~km}^{2}$, or $42.47 \%$. Under RCP8.5, the area decreased by about $46573.50 \mathrm{~km}^{2}$ and $59.24 \%$. Hot spots with highly suitable habitat distribution cover only about $13,832.70 \mathrm{~km}^{2}$. In high-altitude areas, such as eastern Tibet, a new high-altitude suitable habitat appeared, and in highlatitude areas, such as northeastern Shandong Province, a new high-altitude suitable habitat also appeared.

The geometric centroids of the highly suitable habitat of Chinese Beech under the three climatic conditions were calculated, and change were observed. Compared with the geometric center of mass under the current climatic conditions, the geometric center of Chinese Beech moved by about $284.66 \mathrm{~km}$ northwest in the RCP2.6 scenario in 2070 and by about $472.81 \mathrm{~km}$ northwest in the RCP8.5 scenario in 2070 as shown in Fig. 5.

The ecological niche view holds that species or populations occupy relatively stable ecosystem positions and relationships with other populations over a certain time and space [39]. This stability may be broken when environmental factors change. From small species to large ecosystems, the organizational structure and functions will change to adapt to changes in the environment. The plants are sensitive to global climate change [40].

Zhang studied the potential distribution area of Pinus massoniana under projected climate change, indicating that the distribution area of $P$. massoniana will gradually migrate to the north [41]. Li predicted by the model that the suitable distribution area of Larix gmelinii may vacate China in 2100 [42]. Liu simulated the distribution pattern of fir plants in the future climate change scenario and found that the suitable habitat of fir plants moved northward [43]. All of these are consistent with the conclusion that the suitable habitat will move northward under the different climate change scenarios in the future.

Table 4. Changes in the area and center of mass of Chinese Beech.

\begin{tabular}{|c|c|c|c|c|c|}
\hline Type & Area $\left(\mathrm{km}^{2}\right)$ & $\begin{array}{c}\text { Reduced compared with } \\
\text { the present } \\
\left(\mathrm{km}^{2}\right)\end{array}$ & $\begin{array}{c}\text { Less than the present } \\
\%\end{array}$ & $\begin{array}{c}\text { Direction of } \\
\text { change relative to the } \\
\text { present center of mass }\end{array}$ & $\begin{array}{c}\text { Distance } \\
\text { to present center } \\
\text { of mass }(\mathrm{km})\end{array}$ \\
\hline Present & 78615.60 & 0.00 & 0.00 & & \\
\hline RCP2.6 & 45228.70 & 33386.90 & 42.47 & northwest & 284.66 \\
\hline RCP8.5 & 32042.10 & 46573.50 & 59.24 & northwest & 472.81 \\
\hline
\end{tabular}




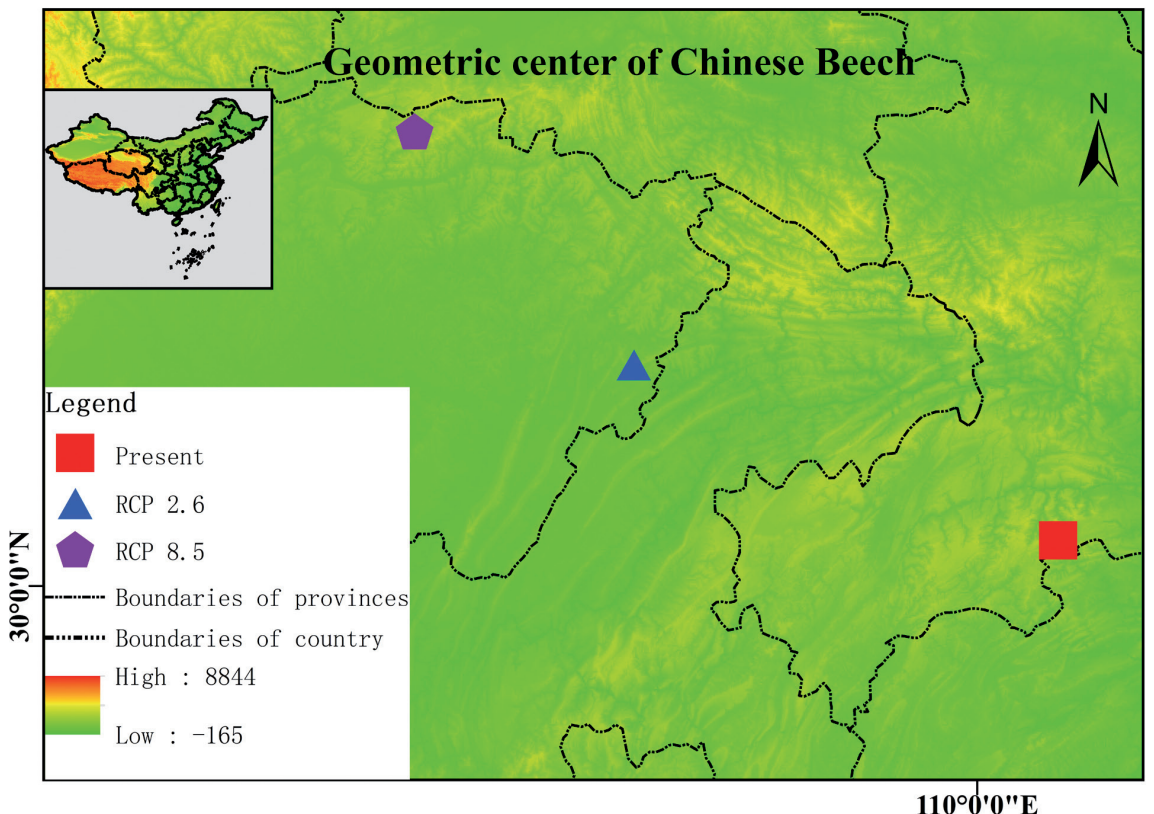

Fig. 5. Geometric center of mass of 7 species Chinese Beech highly suitable habitat under various climatic conditions.

\section{Protection Efficiency}

A spatial analysis was carried out using the intersection tool and by overlaying the vector boundaries of national protected areas as shown in Figs 6-8. The results showed that the current highly suitable habitat area of Chinese Beech is about $78615.60 \mathrm{~km}^{2}$, and only about $9527.36 \mathrm{~km}^{2}$ is located in the protected area, for a protection rate of $12.12 \%$. Under the climate of RCP2.6 in 2070, the highly suitable habitat area of Chinese Beech is about $45228.70 \mathrm{~km}^{2}$, and about $7165.14 \mathrm{~km}^{2}$ is located in the protected area, for a protection rate of $15.84 \%$. Under the climate of RCP8.5 in 2070, the highly suitable habitat area of Chinese Beech is about $32,042.10 \mathrm{~km}^{2}$, and about $4470.96 \mathrm{~km}^{2}$ is located in the protected area, for a protection rate of $13.95 \%$. The hot spot area of Chinese Beech is about $13,832.70 \mathrm{~km}^{2}$, and about $1,683.06 \mathrm{~km}^{2}$ is located in the protected area, for a protection rate of $12.17 \%$. Overall, the protection rate of Chinese Beech is low (Table 5).

In recent years, the survival and development of Chinese Beech are faced with difficulties such as seed dispersal, serious man-made damage and extensive

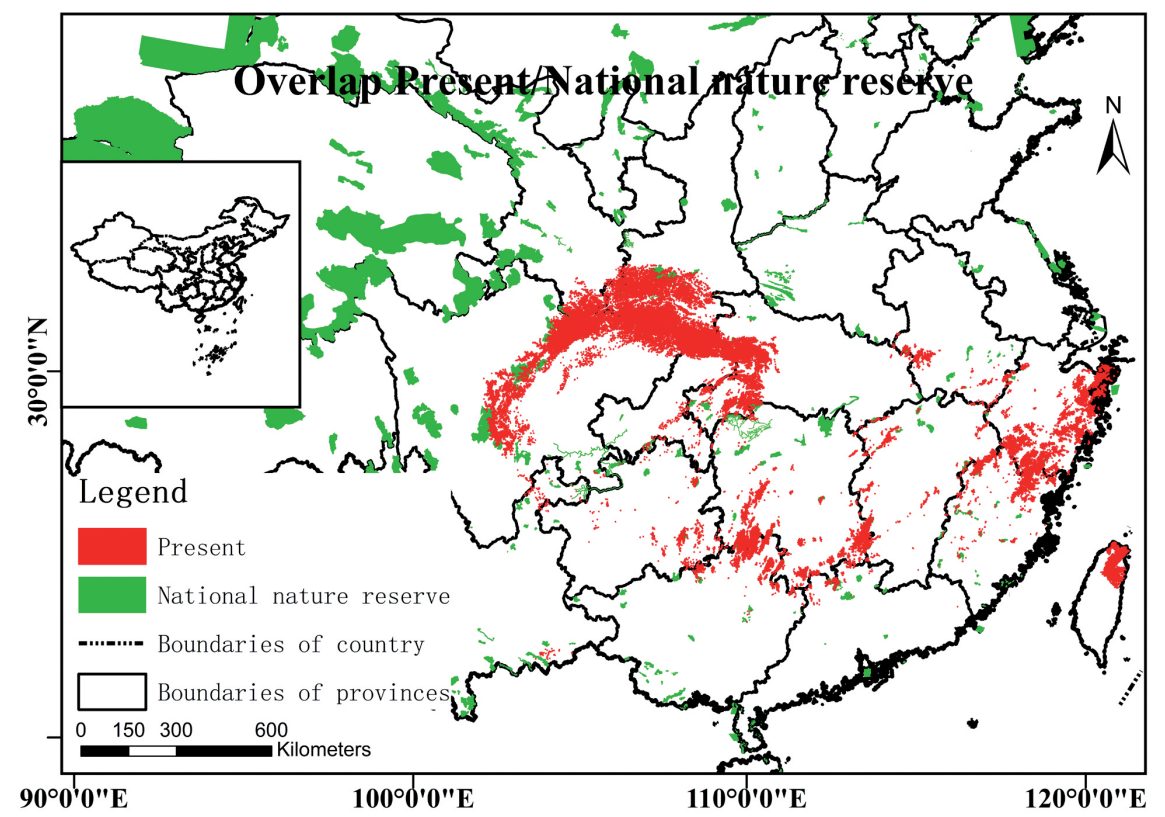

Fig. 6. Protected area of 7 species of Chinese Beech highly suitable habitat under the current climatic conditions. 


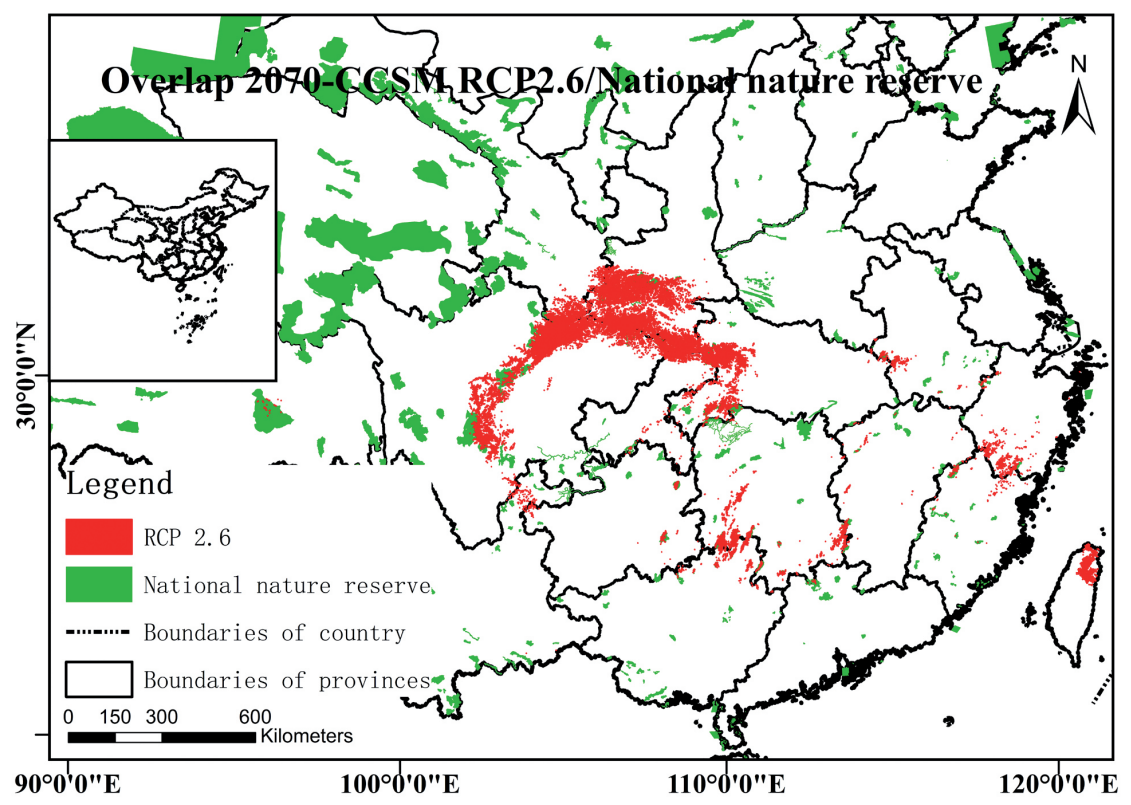

Fig. 7. Protected area of 7 species of Chinese Beech highly suitable habitat under the RCP2.6 climate scenario in 2070.

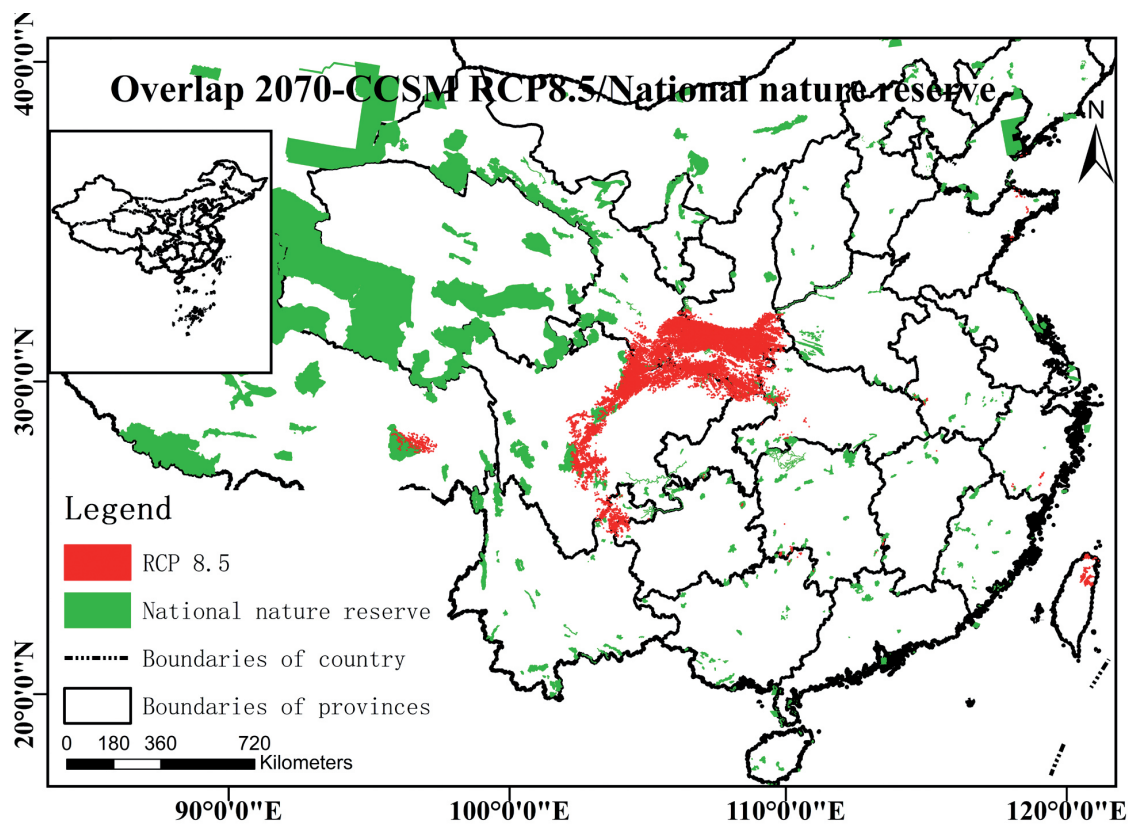

Fig. 8. Protected area of 7 species of Chinese Beech highly suitable habitat under the RCP8.5 climate scenario in 2070.

Table 5. Area and protection rate of Chinese Beech.

\begin{tabular}{|c|c|c|c|c|c|}
\hline Type & Area $\left(\mathrm{km}^{2}\right)$ & Protected area $\left(\mathrm{km}^{2}\right)$ & $\begin{array}{c}\text { Protection rate } \\
(\%)\end{array}$ & $\begin{array}{c}\text { Reduced Compared with } \\
\text { the present }\left(\mathrm{km}^{2}\right)\end{array}$ & $\begin{array}{c}\text { Less than the Present } \\
(\%)\end{array}$ \\
\hline Present & 78615.60 & 9527.36 & 12.12 & 0 & 2362.22 \\
\hline RCP2.6 & 45228.70 & 7165.14 & 15.84 & 5056.40 & 24.79 \\
\hline RCP8.5 & 32042.10 & 4470.96 & 13.95 & & 53.07 \\
\hline Hot spots & 13832.70 & 1683.06 & 12.17 & & \\
\hline
\end{tabular}


destruction of old trees due to global climate change, long-term interference of human activities and weak self-renewal ability of population [18, 38]. Our results showed that, with the continuous increase of greenhouse gas emissions and the continuous warming of climate and environment, the hot spot area of Chinese Beech was only $13,832.70 \mathrm{~km}^{2}$, and the highly suitable habitat area will constantly decrease and the protection rate is low. The highly suitable habitat of Chinese Beech would be reduced by $59.24 \%$, which corresponds to a protection rate of only $13.95 \%$. The above research suggested that a large range of suitable areas of Chinese Beech would be unprotected for a long time, and these areas were very conducive to the distribution and growth of the plant, so these areas should be paid enough attention in the future protection planning.

Although Chinese Beech is likely to migrate to the north and high-altitude areas in response to climate change, we doubt whether Chinese Beech will be able to migrate to these new highly habitable habitats by 2070 from the perspective of actual natural migration. Therefore, it is necessary to consider strengthening the protection of Chinese Beech. Next, the natural migration rate of Chinese Beech can be investigated to identify whether a gap occurs between its actual and theoretical potential. In addition, we can try to reconstruct an artificial population of Chinese Beech in the most suitable habitat in the future, so that it can be more effectively protected.

\section{Conclusion}

Trees from Fagus genus are of ecological and economical importance in China. The projected climate change will apparently impact its distribution, growth and development in the future. Therefore, to have a better understand of the potential geographic distribution of Chinese Beech is particularly important for forest ecosystem and environmental protection. In the present study, precipitation of the driest quarter, minimum temperature of the coldest month and mean diurnal range were the top three most important climatic factors determining the distribution of Chinese Beech, both under the current conditions and different climate scenarios. In the future, a decrease of $59.24 \%$ of the highly suitable area will likely happen due to climate change. The highly suitable habitat was projected to move northwestward and to high altitude areas. On the other hand, currently only $12.12 \%$ of the total area is protected, which could be increased in the future. The present study highlights the fundamental impacts of climate change on tree species distribution and forests ecosystem, and provides valuable information for forests management.

\section{Acknowledgments}

This work was financially supported by the Science \& Technology Department of Sichuan Province (2019YJ0495), the National economy research center project in Western China (CWEER201808), the Education Department of Sichuan Province [18ZA0266]; Mian Yang Normal University (XYCCX201802).

\section{Conflicts of Interest}

The authors declare that they have no conflicts of interest.

\section{Data Availability}

The datasets generated during and/or analyzed during the current study are available from the corresponding author on reasonable request.

\section{References}

1. HU X.G., JIN Y.Q., WANG X.R., MAO J.F., LI Y. Predicting impacts of future climate change on the distribution of the widespread conifer Platycladus orientalis. Plos One, 10, e132326, 2015.

2. CALVIN K., MIGNONE B.K., KHESHGI H.S., SNYDER A.C., PATEL P., WISE M., CLARKE L.E., EDMONDS J. Global market and economic welfare implications of changes in agricultural yields due to climate change. Clim. Chang. Econ., 11, 2050005, 2020.

3. ZOU J., TENG F., FU S. The Latest Progress in Socioeconomic Assessment of the Mitigation of Climate Change-Review of the IPCC Fifth Assessment WGIII Report. Adv. Clim. Chang. Res., 10, 313, 2015 [In Chinese].

4. ABOTSI, K.E., BOSE, R., ADJOSSOU, K., DEBLAUWE, V., KOUAMI, K. Ecological drivers of pteridophyte diversity and distribution in Togo (West Africa). Ecol. Indic., 108, 105741, 2020.

5. BARBER V.A., JUDAY G.P., FINNEY B.P. Reduced growth of Alaskan white spruce in the twentieth century from temperature-induced drought stress. Nature, 405, 668, 2000.

6. BICKFORD D.P., SHERIDAN J.A., HOWARD S.D. Climate change responses: forgetting frogs, ferns and flies? Trends. Ecol. Evol., 26, 553, 2011.

7. MACLEAN L.M.D., WILSON R.J. Recent ecological responses to climate change support predictions of high extinction risk. P. Natl. Acad. Sci. Usa., 108, 12337, 2011.

8. WEI B., WANG R.L., HOU K., WANG X.Y., WU W. Predicting the current and future cultivation regions of Carthamus tinctorius L. using MaxEnt model under climate change in China. Glob. Ecol. Conserv., 16, e477, 2018.

9. WANG C., SHI X.Y., LIU J.G., ZHAO J.C., BO X.Z., CHEN F., CHU Q.Q. Interdecadal variation of potato climate suitability in China. Agr. Ecosyst. Environ., 310, 107293, 2021. 
10. CAI Q., WELK E., JI C., FANG W., SABATINI F.M., ZHU J., ZHU J., TANG Z., ATTORRE F., CAMPOS J.A., ČARNI, A., CHYTRÝ M., ÇOBAN S., DENGLER J., DOLEZAL J., FIELD R., FRINK J.P., GHOLIZADEH H., INDREICA A., JANDT U., KARGER D.N., LENOIR J., PEET R.K., PIELECH R., De SANCTIS M., SCHRODT F., SVENNING J.C., TANG C.Q., TSIRIPIDIS I., WILLNER W., YASUHIRO, K., FANG, J. The relationship between niche breadth and range size of beech (Fagus) species worldwide. J. Biogeogr., 48, 1240, 2021.

11. CAI Q., JI C.J., ZHOU X.L., BRUELHEIDE H., FANG W.J., ZHENG T.L., ZHU J.L., SHI L., LI H.B., ZHU J.L. Changes in carbon storages of Fagus forest ecosystems along an elevational gradient on Mt.Fanjingshan in Southwest China. J. Plant Ecol., 2, 139, 2020.

12. WANG L.N., JIANG X.L., LEI Y., ZHANG M.L. A panbiogeographical explanation of the disjunct distribution of Fagus (Fagaceae) in the northern temperate zone. Chin. J. Plant Ecol., 36, 393, 2012 [In Chinese].

13. TANG L.L., WANG R.X., HE K.S., SHI C., YANG T., HUANG Y.P., ZHENG P.F., SHI F.C. Throwing light on dark diversity of vascular plants in China: predicting the distribution of dark and threatened species under global climate change. PeerJ, 7, e6731, 2019.

14. WU Q.Q., LIANG Z.S., LIU J.L., WENG D.M., ZHANG H.W., YU M.J., HU G. Dynamics of phylogenetic structure in Fagus hayatae communities vary across temporal and spatial scales. Acta Ecol. Sin., 38, 1320, 2018.

15. ZHOU X.L., CAI Q., XIONG X.Y., FANG W.J., ZHU J.X., ZHU J.L., FANG J.Y., JI C.J. Ecosystem carbon stock and within-system distribution in successional Fagus lucida forests in Mt. Yueliang, Guizhou, China. Chin. J. Plant Ecol., 42, 703, 2018 [In Chinese].

16. GE J.L., XIONG G.M., DENG L.Q., ZHAO C.M., SHEN G.Z., XIE Z.Q. Community dynamics of a montane Fagus engleriana-Cyclobalanopsis multiervis mixed forest in Shennongjia, Hubei, China. Biodiversity Sci., 20, 643.

17. RUMBLEA L., TAYLORB G., GRINATHA J.B., MORRISA A.B. Measuring spatial and temporal shifts in forest structure and composition in high elevation beech forests in response to beech bark disease in Great Smoky Mountains National Park. For. Ecol. Manage., 461, 117954, 2020.

18. LI D.D., DONG T.F., CHEN J., SHI Q.M., HE X.X., ZHANG S.P., WU D.J., XU X. Characteristics of Fagus hayatae community and species diversity in Micangshan Nature Reserve. Acta Bot. Boreali-Occident. Sin., 36, 174, 2016.

19. QIN A.L., LIU B., GUO Q.S., BUSSMANN R.W., MA F.Q., JIAN Z.J., XU G.X., PEI S.X. Maxent modeling for predicting impacts of climate change on the potential distribution of Thuja sutchuenensis Franch., an extremely endangered conifer from southwestern China. Glob. Ecol. Conserv., 10, 139, 2017.

20. LI G.Q., XU G.H., GUO K., DU S. Geographical boundary and climatic analysis of Pinus tabulaeformis in China: Insights on its afforestation. Ecol. Eng., 86, 75, 2016.

21. KUMAR S., STOHLGREN T.J. Maxent modeling for predicting suitable habitat for threatened and endangered tree Canacomyrica monticola in New Caledonia. Afr. J. Ecol. Ecos., 6, 1, 2019.

22. GARCIA K., LASCO R., INES A., LYON B., PULHIN F. Predicting geographic distribution and habitat suitability due to climate change of selected threatened forest tree species in the philippines. Appl. Geogr., 44, 12, 2013.
23. PHILliPS S.J., DUDÍK M. Modeling of species distributions with Maxent: new extensions and a comprehensive evaluation. Ecography, 31, 161, 2008.

24. HU J.Y. Study on the Eeological Characteristics of Natural and Secondary Forest of Fagus pashanica. D Type, Sichuan Agricultural University, Chengdu, 2009.

25. HIJMANS R.J., CA MERON S.E., PARRA J.L., JONES P.G., JARVIS A. Very high resolution interpolated climate surfaces for global land areas. Int. J. Cllmatol., 25, 1965, 2010.

26. DROUET L., TAVONI M., BOSETTI V. Selection of climate policies under the uncertainties in the Fifth Assessment Report of the IPCC. Nature Climate Change, 10, 937, 2015.

27. YANG X.Q., KUSHWAHA S.P.S., SARAN S., XU J.C., ROY P.S. Maxent modeling for predicting the potential distribution of medicinal plant, Justicia adhatoda L. in Lesser Himalayan foothills. Ecol. Eng., 51, 83, 2013.

28. LIU L., GUAN L., ZHAO H., HUANG Y., HU J. Modeling habitat suitability of Houttuynia cordata Thunb (Ceercao) using MaxEnt under climate change in China. Ecol. Inform., 63, 101324, 2021.

29. WANG R.L., YANG H., WANG M.T., ZHANG Z., LI Q. Predictions of potential geographical distribution of Diaphorina citri (Kuwayama) in China under climate change scenarios. Sci. Rep., 10, 1, 2020.

30. KAKY E., NOLAN V., ALATAWI A., GILBERT F. A comparison between Ensemble and MaxEnt species distribution modelling approaches for conservation: A case study with Egyptian medicinal plants. Ecol. Inform., 60, 101150, 2020.

31. KOGO B.K., KUMAR L., KOECH R., KARIYAWASAM C.S. Modelling climate suitability for rainfed maize cultivation in Kenya using a maximum entropy (MaxENT) approach. Agronomy, 9, 727, 2019.

32. LUAN X.F., HUANG W.N., WANG X.L., LIU M.C. Identification of hotspots and gaps for biodiversity conservation in Northeast China based on a systematic conservation planning methodology. Acta Ecologica Sinica, 29, 144, 2009 [In Chinese].

33. PETITPIERRE B., KUFFER C., BROENNIMANN O., RANDIN C. Climatic niche shifts are rare among terrestrial plant invaders. Science, 335, 1344, 2012.

34. ELITH J., GRAHAM C.H., ANDERSON R.P., DUDÍK M., FERRIER S., GUISAN A., HIJMANS R.J., HUETTMANN F., LEATHWICK J.R., LEHMANN A. Novel methods improve prediction of species' distributions from occurrence data. Ecography, 29, 129, 2010.

35. ZHANG H.T., LUO D., MU X.D., XU M., WEI H., LUO J.R., ZHANG J.E., HU Y.C. Predicting the potential suitable distribution area of the apple snail Pomacea canaliculata in China based on multiple ecological niche models. Chin. J. Appl. Ecol., 27, 1277, 2016 [In Chinese].

36. DJAMAN K., KOUDAHE K., DARAPUNENI M., IRMAK S. Chilling and Heat Accumulation of Fruit and Nut Trees and Flower Bud Vulnerability to Early Spring Low Temperatures in New Mexico: Meteorological Approach. Sustainability, 13, 2524, 2021.

37. WANG Z.H., BROWN J., TANG Z.Y., FANG J.Y. Temperature dependence, spatial scale, and tree species diversity in eastern Asia and North America. Proc. Natl. Acad. Sci. U. S. A., 106, 13388, 2009.

38. FANG J.Y., LECHOWICZ M.J. Climatic limits for the present distribution of beech (Fagus L.) species in the world. J. Biogeogr., 33, 1804, 2006. 
39. PAPUGA G., GAUTHIER P., PONS V., FARRIS E., THOMPSON J.D. Ecological niche differentiation in peripheral populations: a comparative analysis of eleven Mediterranean plant species. Ecography, 41, 1650, 2018.

40. GONG X., CHEN Y., WANG T., JIANG X., FENG J. Double-edged effects of climate change on plant invasions: Ecological niche modeling global distributions of two invasive alien plants. Sci. Total Environ., 740, 139933, 2020.

41. ZHANG L., LIU S.R., SUN P.S., WANG T.L. Comparative evaluation of multiple models of the effects of climate change on the potential distribution of Pinus massoniana. Chin. J. Plant Ecol., 35, 1091, 2011 [In Chinese].

42. LI F., ZHOU G.S., CAO M.C. Responses of Larix gmelinii geographical distribution to future climate change: A simulation study. Chin. J. Appl. Ecol., 17, 2255, 2006 [In Chinese].

43. LIU R., WANG C.J., HE J., ZHANG Z.X. Analysis of geographical distribution of Abies in China under climate change. Bull. Bot. Res., 38, 37, 2018 [in Chinese]. 